\title{
Transferência de nitrogênio e fósforo para águas superficiais em uma bacia hidrográfica com agricultura e produção pecuária intensiva no Sul do Brasil
}

\author{
Transfer of nitrogen and phosphorus to surface water in a watershed \\ with agriculture and intensive livestock production in Southern Brazil
}

\author{
Viviane Capoane ${ }^{\mathrm{I}}$ Tales Tiecher ${ }^{\mathrm{II}}$ Gilmar Luiz Schaefer ${ }^{\mathrm{II}}$ \\ Lucas Henrique Ciotti ${ }^{\text {II }}$ Danilo Rheinheimer dos Santos ${ }^{I I}$
}

\section{- NOTA -}

RESUMO

Neste trabalho, foram avaliadas as concentrações de fósforo $(P)$ solúvel e nitrato nas águas dos canais de drenagem de uma bacia hidrográfica, com agricultura e produção pecuária intensiva do Sul do Brasil, a fim de avaliar os impactos das atividades agropecuárias no ecossistema aquático. Amostras de água foram coletadas em 11 pontos nos canais de drenagem em três periodos distintos. Coletas diárias, por um periodo de 30 dias, foram efetuadas no médio curso e no exutório da bacia hidrográfica. As concentrações de nitrato e $P$ solúvel foram maiores nas áreas mais antropizadas e com granjas de suínos próximas a cursos d'água. Nas coletas diárias, observou-se que as concentrações de P mudaram consideravelmente nos dias de chuva, mas a concentração de nitrato manteve certa regularidade durante os periodos com e sem chuva. As altas concentrações de P solúvel e nitrato encontradas foram atribuidas ao manejo inadequado das atividades agropecuárias desenvolvidas na bacia hidrográfica.

Palavras-chave: dejetos animais, suinocultura intensiva, pressão antrópica, eutrofização.

\section{ABSTRACT}

In order to evaluate the impact of agricultural activities on aquatic ecosystem, the concentration of nitrate and soluble phosphorus $(P)$ in the stream water were assessed in a rural watershed under intensive agriculture and livestock production in southern Brazil. Water samples were collected at 11 points along the drainage system in three different periods. Additionally, daily collections were made during 30 days in the middle and at the outlet of the watershed. Nitrate and soluble P concentrations were higher in the points closer to the more anthropized areas and pig farms. The soluble $P$ concentration changed substantially according to the rainfall events, while nitrate concentration show little change with rainfall regime. The high nitrate and soluble P concentrations were attributed to poor management of the intensive livestock activities undertaken in the watershed.
Key words: animal waste, intensive pig farming, human disturbance, eutrophication.

Dentre as atividades agropecuárias, a produção intensiva de suínos pode ser considerada a atividade que mais impacta os recursos hídricos (KATO et al., 2009). Tradicionalmente, os efluentes líquidos suínos têm sido utilizados como fertilizante nas lavouras, porque contêm consideráveis concentrações de nutrientes. Normalmente, as doses de dejetos aplicadas são calculadas para satisfazerem a demanda de $\mathrm{N}$ para as culturas. Como a razão $\mathrm{N}: \mathrm{P}$ no dejeto líquido de suíno é menor do que a exigida pelas culturas, as doses de $\mathrm{P}$ aplicadas são de duas a cinco vezes maiores do que as necessidades das culturas, o que leva ao acúmulo de $\mathrm{P}$ nas camadas superficiais do solo (GUARDINI et al., 2012). Dessa forma, a aplicação continuada de efluentes suínos em área agrícolas, a uma taxa que excede a exigida pelas culturas aumenta sobremaneira os riscos de perda de P durante eventos pluviométricos.

Devido à sua alta adsorção ao solo, o $\mathrm{P}$ entra em corpos d'água superficiais adsorvidos às partículas de solo erodidas (MILLER \& GARDINER, 1998). Todavia, a aplicação sucessiva de dejetos em altas doses promove a saturação dos sítios de sorção de $\mathrm{P}$ no solo, resultando na alta disponibilidade desse elemento, sendo facilmente transferido à fase solúvel quando esses sedimentos são transferidos aos corpos d'água (KERR et al., 2011). No presente

\footnotetext{
'Departamento de Geografia, Universidade Federal do Paraná (UFPR), 81531-980, Curitiba, PR, Brasil. E-mail: capoane@gmail.com. Autor para correspondência.

"Departamento de Solos, Universidade Federal de Santa Maria (UFSM), Santa Maria, RS, Brasil. 
trabalho, foram determinadas as concentrações de $\mathrm{P}$ solúvel e nitrato nas águas dos canais de drenagem de uma bacia hidrográfica com agricultura e produção pecuária intensiva do Sul do Brasil, a fim de avaliar os impactos que as atividades agropecuárias podem causar no ecossistema aquático.

A bacia hidrográfica $(\mathrm{BH})$ estudada é predominantemente agrícola (1.336 ha), localizada no município de Palmitinho (236000 e 246000 E e 6974000 e 6980000 S, Datum WGS84, zona 22S), Noroeste do Estado do Rio Grande do Sul. O clima da região corresponde ao subtropical muito úmido, com inverno fresco e verão quente, com precipitações entre 1700-1900 mm ao ano, em 110-140 dias de chuva (ROSSATO, 2011). O substrato litológico é composto de basaltos da Formação Serra Geral, Fácie Paranapanema, com relevo ondulado e cotas altimétricas variando de 198 a $513 \mathrm{~m}$. As classes de solos mais expressivas da $\mathrm{BH}$ são Neossolos e Cambissolos (CUNHA et al., 2010). O mapa de uso da terra foi confeccionado utilizando como base uma imagem de satélite disponível no software Google Earth Pro do ano de 2007. A partir de trabalhos de campo, foi feita a atualização do uso da terra para o ano de 2012. O levantamento espacial das fontes pontuais de poluição (granjas de suínos, estrebarias e residências) da $\mathrm{BH}$ do Arroio Caldeirão foi feito utilizando a imagem de satélite e um GPS de navegação (Gpsmap 62s - Garmin).

As amostras de água foram coletadas no fluxo de base em 11 pontos, sendo oito no canal principal (Pr) e três em um tributário (Tr). A localização dos pontos foi disposta a montante, médio curso e foz do canal principal e num tributário, sempre situando os pontos à montante e à jusante das granjas de criações de suínos e outras fontes potenciais de poluição difusa, como lavouras. As coletas foram realizadas em três períodos distintos, em 7 de outubro de 2012, 9 de dezembro de 2012, e 12 de fevereiro de 2013. No mês de março de 2013, foram efetuadas coletas diárias em dois pontos, no exutório $(\operatorname{Pr} 8)$ e no médio curso $(\mathrm{Pr} 4)$. As amostras de água destinadas à determinação do $\mathrm{P}$ solúvel $\left(\mathrm{PO}_{4}{ }^{3-}\right)$ foram filtradas em membrana de celulose com $0,45 \mu \mathrm{m}$ de diâmetro de poro e as destinadas à determinação do nitrato $\left(\mathrm{NO}_{3}^{-}\right)$ foram filtradas em membrana de celulose com $0,22 \mu \mathrm{m}$ de diâmetro de poro. A concentração de P solúvel foi determinada por espectrofotocolorimetria (MAKRIS, 2002). O nitrato foi determinado por cromatografia líquida de alta performance por troca iônica.

Os resultados das análises químicas da água coletada no fluxo de base comprovam que as atividades antrópicas estão afetando a qualidade da água dos arroios. As concentrações de P solúvel e de nitrato foram maiores nos pontos de coleta amostrados em áreas bastante antropizadas, próximas a estrebarias, granjas de suínos e lavouras (Tabela 1). $\mathrm{O} \operatorname{Pr} 4$ apresentou as maiores concentrações de $\mathrm{P}$ solúvel e nitrato nas três coletas efetuadas, inclusive ultrapassando o limite de $10 \mathrm{mg} \mathrm{L}^{-1}$ de nitrato estabelecido pela Resolução Conama $\mathrm{n}^{\circ} 357$, na segunda coleta (Tabela 1). Este ponto encontra-se logo abaixo de duas granjas de suínos, com lavouras margeando o curso d'água. O Pr1, que é o ponto com menor influência antrópica com matas ciliares bem preservadas, localizado próximo à nascente do canal principal, apresentou as menores concentrações de $\mathrm{P}$ solúvel e nitrato (Tabela 1).

À medida que os arroios passam por áreas antropizadas, houve aumento nas concentrações de $\mathrm{P}$ solúvel e nitrato, como pode ser verificado pela correlação positiva entre a área de lavoura, número de suínos, número de vacas e número de vacas por área de captação com o teor de $\mathrm{P}$ solúvel, e a correlação positiva entre a área de lavoura, número de suínos, número de vacas e número de vacas e suínos por área de captação com o teor de nitrato, na primeira época de amostragem (Tabela 1). Já a relação da área de lavoura pela área total de captação não apresentou relação com as concentrações de $\mathrm{P}$ solúvel e nitrato em nenhuma das coletas. Na segunda e terceira coletas, não houve correlação entre esses parâmetros e os teores de $\mathrm{P}$ solúvel e nitrato. Isso pode ter ocorrido devido às baixas precipitações durante as duas últimas coletas, que diminuiu sobremaneira o escoamento superficial e subsuperficial da $\mathrm{BH}$, que são os principais vetores de transporte de nutrientes aos cursos d'água. Na primeira e segunda coletas, o teor de P solúvel apresentou correlação negativa com a distância média das granjas de suíno ao rio (Tabela 1). Isso ocorre porque o P é um elemento pouco móvel no solo. Dessa forma, quanto mais distantes dos rios estiverem as granjas de suínos e as esterqueiras, menor a chance de transferência desse elemento aos corpos d'água. Já o nitrato, que é um elemento móvel no solo, não apresentou correlação com a distância média das granjas de suínos ao rio.

Assim como observado nas três amostragens realizadas no fluxo de base dos arroios, a água coletada diariamente no mês de março de 2013 no exutório (Pr8) e no médio curso ( $\operatorname{Pr} 4)$, mostrou que, em áreas com intensa atividade antrópica $(\operatorname{Pr} 4)$, as concentrações de $\mathrm{P}$ solúvel e nitrato são altas e, à medida que vai entrando água de tributários menos impactados, há uma diluição nas suas concentrações (Pr8). O nitrato é um elemento bastante móvel, devido à sua baixa carga residual e por isso, em dias 
Tabela 1 - Caracterização dos pontos de monitoramento e teores de fósforo solúvel e nitrato em três épocas, na bacia hidrográfica do Arroio Caldeirão, Palmitinho, Rio Grande do Sul.

\begin{tabular}{|c|c|c|c|c|c|c|c|}
\hline $\begin{array}{l}\text { Pontos (identificação } \\
\text { das granjas de suínos) }\end{array}$ & $\begin{array}{c}\text { Área de } \\
\text { captação (ha) }\end{array}$ & Lavoura (ha) & Lavoura (\%) & $\mathrm{N}^{\mathrm{o}}$ de suínos & $\begin{array}{c}\mathrm{N}^{\mathrm{o}} \text { de } \\
\text { Suínos/área } \\
\text { captação }\end{array}$ & $\mathrm{N}^{\mathrm{o}}$ de vacas & $\begin{array}{c}\mathrm{N}^{\mathrm{o}} \mathrm{de} \\
\text { vacas/área } \\
\text { captação }\end{array}$ \\
\hline Pr 1 (nenhuma) & 11 & 5 & 45,5 & 0 & 0.0 & 0 & 0.0 \\
\hline $\operatorname{Pr} 2(8,9,10)$ & 230 & 84 & 36,5 & 1550 & 6.7 & 100 & 1.2 \\
\hline $\operatorname{Pr} 3(5$ a 11$)$ & 474 & 204 & 43,0 & 4550 & 9.6 & 194 & 1.0 \\
\hline $\operatorname{Pr} 4(3$ a 11,13$)$ & 639 & 271 & 42,4 & 5917 & 9.3 & 250 & 0.9 \\
\hline $\operatorname{Pr} 5(3$ a $11,13,15)$ & 799 & 338 & 42,3 & 5990 & 7.5 & 299 & 0.9 \\
\hline $\operatorname{Pr} 6(3$ a 13,15$)$ & 966 & 405 & 41,9 & 6490 & 6.7 & 385 & 1.0 \\
\hline $\operatorname{Pr} 7(2$ a 13,15$)$ & 1143 & 471 & 41,2 & 7310 & 6.4 & 469 & 1.0 \\
\hline $\operatorname{Pr} 8(1$ a 15$)$ & 1333 & 521 & 39,1 & 8221 & 6.2 & 516 & 1.0 \\
\hline Tr 1 (nenhuma) & 10 & 4 & 40,0 & 0 & 0.0 & 0 & 0.0 \\
\hline $\operatorname{Tr} 2(11)$ & 71 & 33 & 46,5 & 700 & 9.9 & 20 & 0.6 \\
\hline $\operatorname{Tr} 3(6,7,11)$ & 97 & 47 & 48,5 & 2200 & 22.7 & 41 & 0.9 \\
\hline $\begin{array}{l}\text { Pontos (identificação } \\
\text { das granjas de suínos) }\end{array}$ & $\begin{array}{l}\text { Distância } \\
\text { média das } \\
\text { granjas ao rio } \\
\text { (m) }\end{array}$ & -------- Fós & oro solúvel (ms & $\left.L^{-1}\right)$------------ & 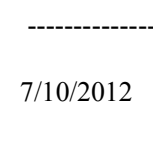 & Nitrato $\left(\mathrm{mg} \mathrm{L}^{-1}\right.$ & ----------- \\
\hline Pr 1 (nenhuma) & - & 0,006 & - & - & 1,22 & - & - \\
\hline $\operatorname{Pr} 2(8,9,10)$ & $115 \pm 34$ & 0,015 & 0,021 & 0,017 & 2,22 & 9,39 & 0,84 \\
\hline $\operatorname{Pr} 3(5$ a 11$)$ & $98 \pm 41$ & 0,013 & 0,023 & 0,001 & 2,78 & 4,94 & 0,47 \\
\hline $\operatorname{Pr} 4(3$ a 11,13$)$ & $90 \pm 42$ & 0,020 & 0,045 & 0,027 & 3,35 & 14,81 & 2,72 \\
\hline $\operatorname{Pr} 5(3$ a $11,13,15)$ & $87 \pm 41$ & 0,019 & 0,038 & 0,025 & 2,65 & 5,35 & 0,86 \\
\hline $\operatorname{Pr} 6(3$ a 13,15$)$ & $97 \pm 51$ & 0,023 & 0,037 & 0,021 & 2,77 & 4,15 & 0,52 \\
\hline $\operatorname{Pr} 7(2$ a 13,15$)$ & $94 \pm 50$ & 0,024 & 0,032 & 0,026 & 2,98 & 4,01 & 0,74 \\
\hline $\operatorname{Pr} 8(1$ a 15$)$ & $95 \pm 49$ & 0,021 & 0,024 & 0,011 & 3,31 & 7,20 & 0,27 \\
\hline $\operatorname{Tr} 1$ (nenhuma) & - & 0,010 & - & - & 1,47 & - & - \\
\hline $\operatorname{Tr} 2(11)$ & 134 & 0,011 & - & - & 2,85 & - & - \\
\hline $\operatorname{Tr} 3(6,7,11)$ & $92 \pm 53$ & 0,013 & - & - & 3,82 & - & - \\
\hline \multicolumn{2}{|l|}{ Área de lavoura (ha) } & $\begin{array}{l}\text { es de fósforo } \\
0.91 * * * *\end{array}$ & $\begin{array}{l}\text { ivel e nitrato } \\
\text { ns }\end{array}$ & $\begin{array}{l}\text { as caracte } \\
\text { ns }\end{array}$ & $\begin{array}{l}\text { s dos pont } \\
0.47^{*}\end{array}$ & $\begin{array}{c}\text { nostrados } \\
\text { ns }\end{array}$ & ns \\
\hline \multicolumn{2}{|l|}{ Área de lavoura (\%) } & ns & ns & ns & $0.82 * * *$ & ns & ns \\
\hline \multicolumn{2}{|l|}{$\mathrm{N}^{\mathrm{o}}$ de suínos } & $0.92 * * * *$ & ns & ns & $0.60 *$ & ns & ns \\
\hline \multicolumn{2}{|c|}{$\mathrm{N}^{0}$ de suínos/área captação } & ns & ns & ns & ns & ns & ns \\
\hline \multicolumn{2}{|l|}{$\mathrm{N}^{\mathrm{o}}$ de vacas } & $0.92 * * * *$ & ns & ns & $0.47 *$ & ns & ns \\
\hline \multicolumn{2}{|c|}{$\mathrm{N}^{\mathrm{o}}$ de vacas/área captação } & $0.73 * *$ & ns & ns & $0.73 * * *$ & ns & ns \\
\hline \multicolumn{2}{|c|}{ Distância média das fontes ao rio } & $-0.59 *$ & $-0.70^{*}$ & ns & ns & ns & ns \\
\hline
\end{tabular}

ns, não significativo; * significativo a $10 \%$ de probabilidade; $* *$ significativo a $5 \%$ de probabilidade; $* * *$ significativo a $1 \%$ de probabilidade; $* * * *$ significativo a $0,1 \%$ de probabilidade.

de chuva, este elemento é rapidamente lixiviado. Isto pode ser observado no ponto de coleta no médio curso (Figura 1). No exutório, as concentrações de nitrato foram superiores nos dias de chuva, o que decorre dos fluxos d'água das áreas a montante. As concentrações de nitrato nos pontos monitorados variaram de 0,1 a $5,6 \mathrm{mg} \mathrm{L}^{-1}$ no médio curso e de 0,1 a $4,2 \mathrm{mg} \mathrm{L}^{-1}$ no exutório (Figura 1). O P solúvel apresentou os maiores picos nas coletas realizadas próximo aos eventos pluviométricos (Figura 1). Isso se deve à alta energia de ligação desse elemento às partículas de solo e, por isso, a concentração de fosfato nos canais de drenagem da BH está relacionada ao escoamento superficial de águas pluviais. As concentrações de $\mathrm{P}$ solúvel variaram de 0,013 a $0,178 \mathrm{mg} \mathrm{L}^{-1}$ no ponto localizado no médio curso da $\mathrm{BH}$ e de 0,013 a $0,105 \mathrm{mg} \mathrm{L}^{-1}$ no exutório. Nos dias 4 e 12 de março, as concentrações de $\mathrm{P}$ ultrapassaram até mesmo o limite de $0,15 \mathrm{mg}$ $\mathrm{L}^{-1}$ de $\mathrm{P}$ total, para enquadramento na classe 3 do Conama $n^{\circ} 357$. Geralmente, concentrações na faixa de $0,01 \mathrm{mg} \mathrm{L}^{-1}$ de P são suficientes para manutenção do fitoplâncton, e concentrações na faixa de 0,03 a $0,10 \mathrm{mg} \mathrm{L}^{-1}$, ou maiores, podem desencadear o seu crescimento desenfreado (USEPA, 1996). 


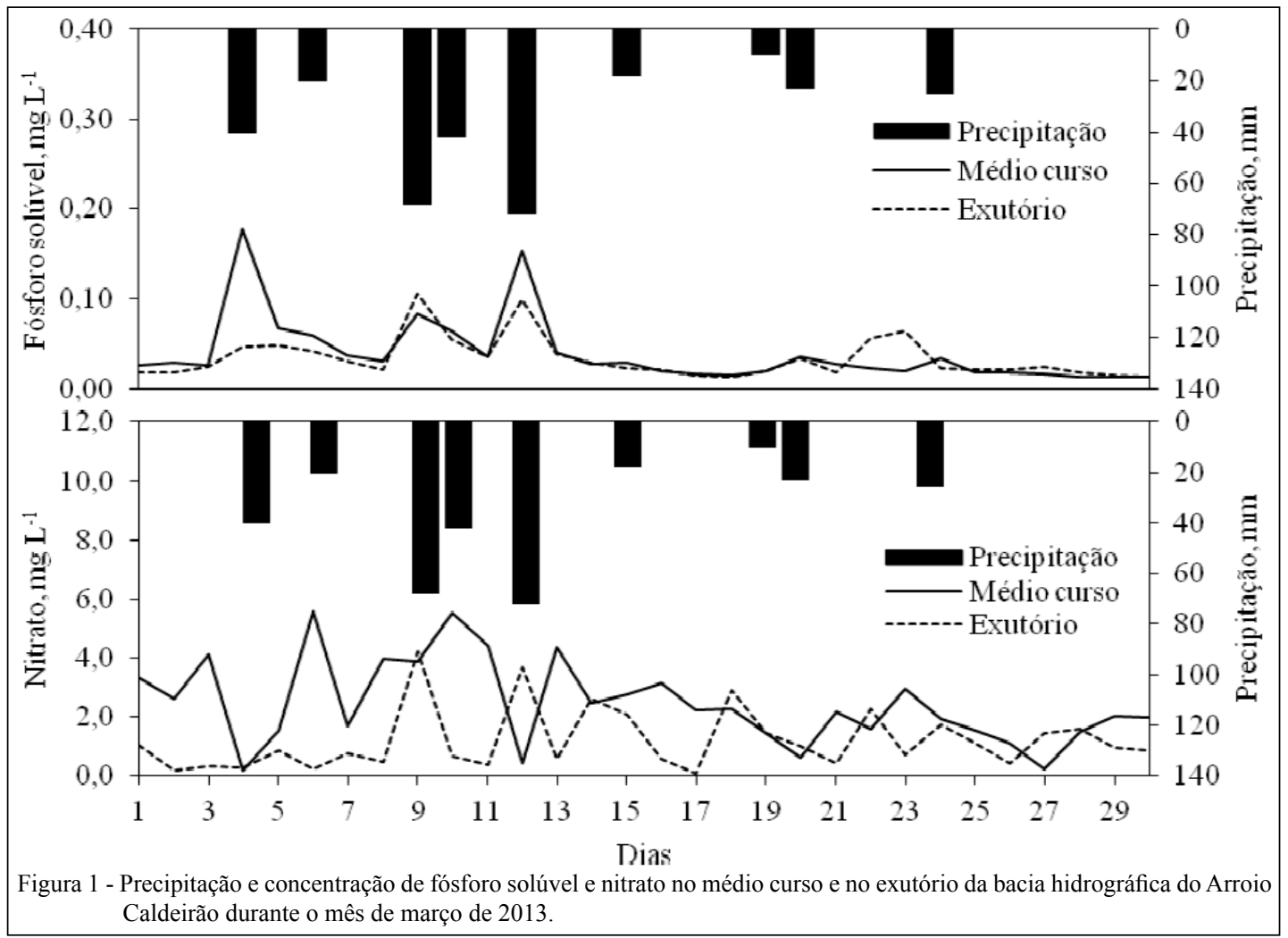

Conclui-se, portanto, que as atividades agropecuárias desenvolvidas na bacia estão degradando a qualidade das águas superficiais. Como as redes fluviais ligam paisagens terrestres, as perturbações nesse ecossistema podem ter grandes consequências nos ciclos biogeoquímicos em escalas local, regional e global. Nesse sentido, é importante estabelecer práticas de gestão adequadas, tanto nas áreas agrícolas como nas estrebarias e granjas de suínos, a fim de evitar perdas de solo, nutrientes e poluentes para os sistemas aquáticos.

\section{AGRADECIMENTOS}

Os autores agradecem à Fundação de Amparo à Pesquisa do Estado do Rio Grande do Sul (FAPERGS), Conselho Nacional de Desenvolvimento Científico e Tecnológico (CNPq) e Coordenação de Aperfeiçoamento de Pessoal de Nível Superior (CAPES), pelo auxílio financeiro e pelas bolsas de Graduação, Mestrado, Doutorado e Produtividade em Pesquisa dos autores.

\section{REFERÊNCIAS}

CUNHA, N. G. et al. Estudos de Solos do Município de Palmitinho, RS. Documentos (Circular Técnica, Embrapa Clima Temperado), v. 105, p. 1-28, 2010.

GUARDINI, R. et al. Accumulation of phosphorus fractions in typic Hapludalf soil after long-term application of pig slurry and deep pig litter in a no-tillage system. Nutrient Cycling in Agroecosystems, v. 93, n. 2, p. 215-225, 2012. Disponível em: <http://link.
springer.com/article/10.1007\%2Fs10705-012-9511-3\#page-1>. Acesso em: 29 jul. 2014. doi: 10.1007/s10705-012-9511-3.

KATO, T. et al. Runoff characteristics of nutrients from an agricultural watershed with intensive livestock production. Journal of Hydrology, v. 368, n. 1-4, p. 79-87, 2009. Disponível em: < http://www.sciencedirect.com/science/article/pii/ S0022169409000511>. Acesso em: 29 jul. 2014. doi: 10.1016/j. jhydrol.2009.01.028.

KERR et al. Examining the link between terrestrial and aquatic phosphorus speciation in a subtropical catchment: The role of selective erosion and transport of fine sediments during storm events. Water Research, v. 45, n. 11, p. 3331-3340, 2011. Disponível em: <http://www.sciencedirect.com/science/article/pii/ S0043135411001667>. Acesso em: 29 jul. 2014. doi: 10.1016/j. watres.2011.03.048

MAKRIS, K. C. Soil and colloidal phosphorus dynamics in three KY soils: bioavailability, transport and water quality implications. 2002. 170f. Dissertação (Master Science in the College of Agriculture at the University of Kentucky) - College of Agriculture at the University of Kentucky, Lexington.

MILLER, R. W.; GARDINER. D. T. Soils in our environment. 8th ed. Prentice Hall, Upper Saddle River, NJ. 1998.

ROSSATO, M. S. Os climas do Rio Grande do Sul: variabilidade, tendências e tipologia. 2011. 240f. Tese (Doutorado em Geografia) Universidade Federal do Rio Grande do Sul, Porto Alegre, Rio Grande do Sul.

US Environmental Protection Agency (USEPA). Proposed guidelines for ecological risk assessment: Notice. FRL-5605-9. Federal Register, 61, 47552-47631, 1996.

Ciência Rural, v.45, n.4, abr, 2015. 